\title{
Factores de riesgo psicosociales extra laborales y su relación con el estrés y la salud mental - Estudio de Caso con la Empresa Cosmética ${ }^{1}$
}

\author{
María Fernanda Caballero Lozada ${ }^{2}$ \\ Genny Amparo Gallo García ${ }^{3}$ \\ María Isabel Posso ${ }^{4}$ \\ Julio Cesar Montoya R. ${ }^{5}$
}

\section{Resumen}

El presente proyecto muestra un trabajo investigativo en la empresa Cosmética donde se analizaron los factores de riesgo extra laborales y los niveles de estrés con sus patologías. El proceso metodológico consistió en identificación de referentes teóricos, caracterización de la población objeto de estudio, planteamiento del problema, pregunta de investigación, objetivos. Se define la muestra para aplicar las técnicas e instrumentos seleccionados, se sistematiza la información, se analiza e identifica los factores de riesgo Psicosociales Extra laborales, la información sociodemográfica y ocupacional y los niveles de estrés con sus patologías. La información recolectada en la batería de factores de Riesgo Psicosociales del Ministerio de Protección Social (2010), las entrevistas y el ejercicio de observación, evidenciaron que la empresa Cosmética, posee altos niveles de estrés en el $80 \%$ de la población encuestada, los cuales podrían estar relacionados con los factores de riesgo extralaborales que arrojaron niveles altos de riesgo. Se presentaron las siguientes patologías; somnolencia, agotamiento, falta de energía, dolores musculares, problemas

\footnotetext{
${ }^{1}$ El nombre de la Empresa se ha cambiado por razones de confidencialidad.

2 Psicóloga, Magíster en Psicología, estudiante de Doctorado en Psicología, docente Universidad Nacional Abierta y a Distancia. Grupo de investigación: Subjetividades y sujetos colectivos. maria.caballero@unad.edu.co

${ }^{3}$ Administradora de Empresas, Estudiante de Psicóloga de la Universidad Nacional Abierta y a Distancia - UNAD

${ }^{4}$ Psicóloga, Especialista en pedagogía del desarrollo del aprendizaje Autónomo, Magister en Administración de organizaciones. Docente. Universidad nacional Abierta y a Distancia- UNAD. maria.posso@unad.edu.co
}

${ }^{5}$ Administrador de Empresas, Especialista en Pedagogía para el Desarrollo del Aprendizaje Autónomo, Maestría en Administración de Empresas. Investigador asociado del grupo de investigación llama. Docente de la Universidad Nacional Abierta y a Distancia - UNAD. julio.montoya@unad.edu.co 
gastrointestinales, sentimiento de sobrecarga de trabajo, dificultad para concentrarse, cansancio, ganas de no asistir al trabajo, disminución del rendimiento laboral, desinterés y bajo compromiso con lo que hace. La exposición continua a altos niveles de estrés genera desgaste de energía, agotamiento emocional que repercuten en la salud del trabajador.

\section{Introducción}

Los riesgos psicosociales en el trabajo cada vez adquieren más relevancia, dado que las enfermedades mentales generan más ausentismo e incapacidades que los mismos riesgos físicos; según “evidencias encontradas en la relación entre los riesgos psicosociales en el trabajo y el incremento de procesos mórbidos en los trabajadores" (Villalobos, 2004)

La exposición continúa a altos niveles de estrés, genera desgaste de energía y agotamiento emocional que repercuten en la salud del trabajador desarrollando enfermedades físicas como gastritis, reflujo, dolores lumbares y musculares, dolores de cabeza y enfermedades psicosomáticas como sentimientos de ineficacia y baja autoestima, falta de sueño, falta de apetito, apatía, dolores crónicos etc.

En Colombia, el MPS, expidió la resolución 2646 (2008), mediante la cual, "se establecen disposiciones y se definen responsabilidades para la identificación, evaluación, prevención, intervención y monitoreo permanente de la exposición a factores de riesgo psicosociales y para la determinación del origen de las patologías causadas por el estrés ocupacional" (p.1), y además señala que "los factores psicosociales deben ser evaluados objetiva y subjetivamente, utilizando instrumentos que para el efecto hayan sido validados en el país”. (MPS - Batería de instrumentos para la evaluación del riesgo psicosocial, 2010, p.11)

En el presente proyecto, se muestra un trabajo investigativo en una empresa cosmética donde se analizaron los factores de riesgo extra laborales y los niveles de estrés con sus patologías. 


\section{Riesgos Psicosociales}

Gunnar Nerell y C.G. Sandberg (1993), (mencionados por Betancur Gómez, 2013), definen el riesgo psicosocial como:

"la condición o condiciones propias del individuo, medio laboral y del entorno extralaboral que, bajo determinadas condiciones de intensidad y de tiempo de exposición, producen efectos negativos en el trabajador y, por último, estrés ocupacional, el cual puede generar desajustes en la salud del individuo o individuos a nivel intelectual, fisiológico, psico-emocional y social”'(p.28)

De igual forma para Toro, Londoño, Sanín y Valencia (2010), Mencionados por Betancur, 2013) definen los factores psicosociales ocupacionales como. "una condición presente en el trabajo, de carácter tecnológico, organizacional, social, económico o personal, con la que se relaciona un individuo, y que puede afectar positiva o negativamente la salud, el bienestar, el desempeño o el desarrollo personal o colectivo". (p.28). y además consideran que:

"El factor de riesgo psicosocial es cualquier condición psicosocial presente en el trabajo de una persona o colectividad laboral que puede afectar negativamente su salud, bienestar, desempeño y, también, su desarrollo personal. Según ellos, el factor protector psicosocial se presenta cuando dicha condición minimiza o elimina un riesgo y, además, afecta positivamente las dimensiones antes descritas”.(p.28)

\section{Riesgos Psicosociales Extra laborales}

Según el MPS en la batería de instrumentos para la evaluación de los riesgos Psicosociales (2010), las Condiciones Psicosociales Extra laborales, "comprenden los aspectos del entorno familiar, social y económico del trabajador. A su vez, abarcan las condiciones del lugar de vivienda, que pueden influir en la salud y bienestar del individuo" 
(p.27). y están se evalúan a través de siete dimensiones que son: Tiempo fuera del trabajo, relaciones Familiares, comunicación y relaciones Interpersonales, situación económica del grupo familiar, características de la vivienda y su entorno, influencia del entorno extra laboral sobre el trabajo, desplazamiento vivienda - trabajo -vivienda

\section{El trabajo, el estrés y la salud}

Una de las últimas investigaciones a nivel mundial acerca de trabajo, estrés y salud se dio "en los Ángeles en septiembre de 2013, donde más de 39 países se reunieron en la X conferencia Internacional sobre Estrés y Salud Laboral, la cual se enfoca en reducir el estrés y mejorar la salud de los trabajadores" (Weir, 2013, parr.1)

En este encuentro, se ilustra un punto de vista más amplio de la salud en el trabajo, avanzando en la idea de que los factores intra y extra-laborales contribuyen a la salud y la seguridad de la fuerza laboral de hoy; teniendo en cuenta que la salud y el trabajo son importantes en nuestras vidas y van ligadas la una de la otra; se unen estos dos conceptos integrando la seguridad en el trabajo y la promoción de la salud (Casey Chosewood (mencionado por Weir,(2013), además agrega que

"Lo que sucede en el trabajo no se queda en el trabajo, y lo que sucede en el país no se queda en casa." (...) y demás "están invitando a los empleados a tomar decisiones saludables cuando están fuera del trabajo, proporcionando oportunidades de educación para la salud o exámenes médicos etc." Casey Chosewood (mencionado por Weir (2013, parr. 4-6).

\section{Promoción de la salud Mental.}

LaMontagne, (mencionado por Weir, 2013) "establece un acercamiento intervención integrada al trabajo y la salud mental. La ansiedad, depresión y otros problemas de salud mental son frecuentes en las poblaciones de trabajo, y que algunos de esos problemas son atribuibles a las condiciones de trabajo" (parr. 9). 
De igual forma, afirma LaMontagne, que los empleadores, deben tener en cuenta los factores de riesgo relacionados con la salud mental, independientemente de su causa, dado que al final no importa si el empleado está deprimido por causas laborales o por problemas familiares. (parr.12); dado que de todas formas esa patología le va a afectar su rendimiento laboral

\section{Responsabilidad Social Empresarial -RSE}

Los empresarios, tienen la obligación Jurídica de proteger la seguridad y Salud del empleado y también una obligación ética y moral llamada Responsabilidad Social Empresarial con los empleados y la sociedad. Generando bienestar y mejorando la calidad de vida de las personas. Debe protegerlos del estrés y otros riesgos psicosociales pero no solo dentro de la empresa si no en el entorno social y familiar del trabajador.

El origen de la RSE data de los años 50 y 60 en Estados Unidos y se asienta en Europa en los 90, cuando la Comisión Europea utilizó este concepto para involucrar a los empresarios en una estrategia de empleo que generase mayor cohesión social. El proceso de incluir a los empresarios en las soluciones de tipo social estableció la reconciliación entre las empresas y comunidad; tal hecho propició la conexión social, la solidaridad y el respeto al medio ambiente (Publicaciones Vértice, 2009). Mencionado por (Aguilera Acosta, 2012, p.4)

Según Aguilera Acosta (2012) "La Responsabilidad Social Empresarial se define como el compromiso que asumen las empresas hacia la sociedad en beneficio del desarrollo sostenible" (p.5), la cual asumen las empresas voluntariamente, motivados por un interés moral social y económico y tiene que ver con los factores intra y extra laborales.

La RSE es interna y externa; para el caso solo trabajaremos con la RSE fuera del lugar de trabajo dado que en los últimos años los empresarios han visto los beneficios de mejorar el entorno laboral. 
En el caso de El crecimiento de la empresa se ve beneficiado al mejorar la confianza y el compromiso de los empleados, se perfecciona la motivación y se crea la cultura organizacional que propicie la generación de valor agregado en los productos o servicios, la creatividad y la innovación. La calidad de vida de los empleados de la organización debe reflejar el compromiso que tiene la empresa con prácticas responsables que los ayuden a aportar como individuos al mejoramiento de la sociedad. (Aguilera Acosta, 2012, p.20)

Por otro lado, las empresas que se ocupan de brindar a sus empleados un buen ambiente de trabajo tienen menores índices de rotación de personal.

\section{Marco Legal}

En los últimos años se ha dado más importancia a la salud mental de los empleados, dado que la exposición permanente a factores de riesgo psicosocial son los que más accidentes laborales e incapacidades por enfermedades físicas y mentales generan (Cabrera, 2010, p.11), mencionada por el MPS en la Batería de Instrumentos para la evaluación de los Riesgos Psicosociales (2010, p.11)

Los factores de riesgo psicosociales, son condiciones psicológicas que pueden generar efectos negativos en la Salud física y mental del trabajador; se habla de factores de riesgo dado que al exponerse por tiempo prolongado a la condición negativa se puede desarrollar un estrés crónico. De igual forma las condiciones extra laborales contribuyen a la salud mental del trabajo según afirma la OIT/OMS en el Informe de Comité mixto sobre factores psicosociales (1984), "los factores externos al lugar de trabajo, pero que guardan relación con las preocupaciones psicosociales en el trabajo, se derivan de circunstancias familiares o de la vida privada, de elementos culturales, la nutrición, las facilidades de transporte y la vivienda" (p.12)

Así mismo afirma el autor que el tiempo en el lugar de trabajo, cambia la dinámica familiar y social y la forma de vida afectando la salud del trabajador (...) (p.16)., a lo que 
se suma el desempleo y la situación económica, dado que afecta la estabilidad laboral y por ende el bienestar de los trabajadores que aceptan trabajar en condiciones inhumanas con horarios extendidos y una carga laboral alta, colocando al trabajador en una posición débil para enfrentar la carga laboral (...) (p.20). Esta rutina diaria, a mediano plazo, trae consecuencias negativas a nivel psicológico, dado que deteriora la relación con su familia y amigos llegando a casa cansado y de mal genio, lo cual le puede generar insomnio, irritación, preocupación, tensión y depresión; actitudes negativas que transportará de su casa al trabajo derivándose en problemas cognoscitivos como pérdida de capacidad de atención, memoria y toma de decisiones (p.25), generando estrés. (OIT/OMS Informe de Comité mixto sobre factores psicosociales, 1984, p.16-25)

Así pues, la exposición continúa a altos niveles de estrés, genera desgaste de energía y agotamiento emocional que repercuten en la salud del trabajador desarrollando enfermedades físicas como gastritis, reflujo, dolores lumbares y musculares, dolores de cabeza y enfermedades psicosomáticas como sentimientos de ineficacia y baja autoestima, falta de sueño, falta de apetito, apatía, dolores crónicos etc.

En Colombia, el MPS, expidió la resolución 2646 (2008), mediante la cual, “se establecen disposiciones y se definen responsabilidades para la identificación, evaluación, prevención, intervención y monitoreo permanente de la exposición a factores de riesgo psicosociales y para la determinación del origen de las patologías causadas por el estrés ocupacional" (p.1)., y además señala que "los factores psicosociales deben ser evaluados objetiva y subjetivamente, utilizando instrumentos que para el efecto hayan sido validados en el país”. (MPS - Batería de instrumentos para la evaluación del riesgo psicosocial, 2010, p.11)

El Decreto 1832 de agosto 3 de 1994, en su Artículo 1º Tabla de enfermedades profesionales, en el punto No. 42 tipifica las patologías causadas por estrés en el trabajo así: 
Trabajos con sobrecarga cuantitativa, demasiado trabajo en relación con el tiempo para ejecutarlo, trabajo repetitivo combinado con sobrecarga de trabajo. Trabajos con técnicas de producción en masa, repetitiva o monótona o combinada con ritmo o control impuesto por la máquina. Trabajos por turnos, nocturno y trabajos con estresantes físicos con efectos psicosociales, que produzcan estados de ansiedad y depresión, infarto del miocardio y otras urgencias cardiovasculares, hipertensión arterial, enfermedad acido péptica severa o colon irritable. (p.5)

En el 2014 el gobierno nacional según el Decreto 1477, adopta la nueva tabla de enfermedades laborales con el fin de mejorar las condiciones del empleado; sin embargo en esta tabla no se especifica claramente el estrés, (la información es ambigua y generalizada). En la sección I punto 4, para los agentes psicosociales, se señalan los factores de riesgo psicosociales y unas patologías generadas por la exposición a esos riesgos, las cuales incluyen entre otros: gastritis, ansiedad, trastornos emocionales, de comportamiento y de adaptación; síndrome del colon irritable, trastornos del sueño, de igual forma depresión, Infarto del miocardio y otras urgencias cardiovasculares, hipertensión arterial etc. (p.2628).

Aunque no especifica claramente que estas patologías son causadas por estrés, varios estudios a nivel internacional afirman que estas se dan por la exposición continua al estrés; por lo tanto; se deduce que estas patologías son causadas por estrés (Ver anexo 3 Tabla de enfermedades laborales)

\section{Procedimiento}

El proceso metodológico se realizó siguiendo las siguientes etapas: identificación y caracterización de la población objeto de estudio, identificación de los referentes teóricos; planteamiento del problema y pregunta de investigación y los objetivos. Se define la muestra para empezar a aplicar las técnicas e instrumentos seleccionados, seguidamente se sistematiza la información, se analiza e identifica los factores de riesgo Psicosociales Extra 
laborales, la información sociodemográfica y ocupacional y los niveles de estrés con sus patologías.

Después de sistematizar los datos de los cuestionarios, los resultados brutos se transformaron de acuerdo a un factor de transformación definido en los cuestionarios, después de esto, se compara ese resultado con unos baremos dados por el ministerio de protección Social y se correlacionan los factores de riesgo extra laborales con el nivel de estrés, la información sociodemográfica y ocupacional y las patologías presentadas

\section{Grafica 1 Mapa de Proceso Metodológico}

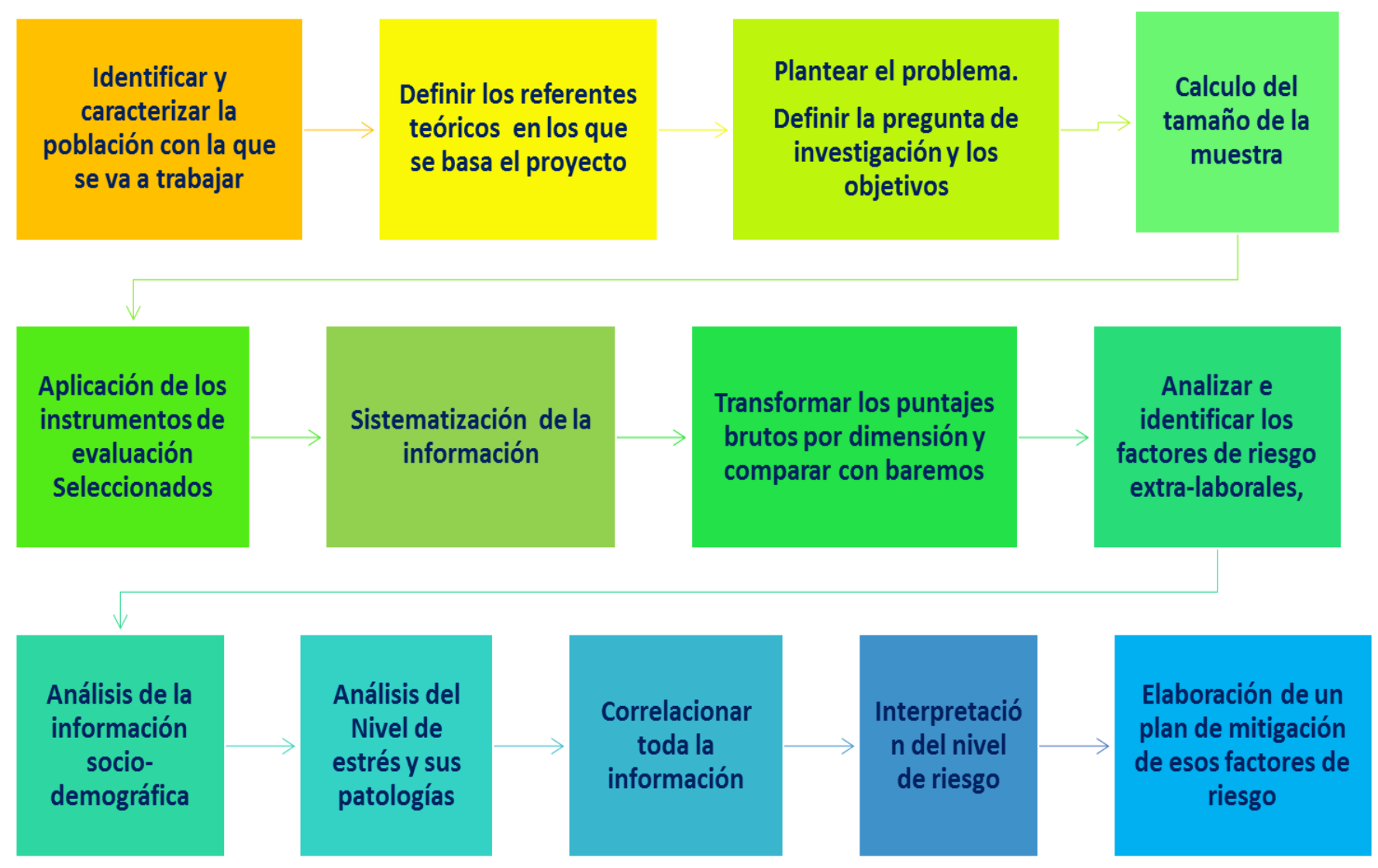

Fuente: Elaboración Propia 


\section{Descripción análisis, interpretación y discusión}

\section{Información sociodemográfica}

\section{Grafica 2}

\begin{tabular}{|c|c|c|c|}
\hline Variables & Categorias & Cant & $\%$ \\
\hline \multirow{4}{*}{ Edad } & De 20 a 30 Años & 1 & $20 \%$ \\
\hline & De 31 a 40 años & 2 & $40 \%$ \\
\hline & De 41 a 50 Años & 2 & $40 \%$ \\
\hline & \begin{tabular}{|l} 
Total \\
\end{tabular} & 5 & $100 \%$ \\
\hline \multirow{3}{*}{ Genero } & Mujer & 2 & $40 \%$ \\
\hline & Hombre & 3 & $60 \%$ \\
\hline & Total & 5 & $100 \%$ \\
\hline \multirow{4}{*}{$\begin{array}{c}\text { Estado } \\
\text { Civill }\end{array}$} & Soltero & 1 & $20 \%$ \\
\hline & Casado & 3 & $60 \%$ \\
\hline & Union Libre & 1 & $20 \%$ \\
\hline & Total & 5 & $100 \%$ \\
\hline \multirow{4}{*}{$\begin{array}{c}\text { Nivel } \\
\text { Estudios }\end{array}$} & Bachiller & & $0 \%$ \\
\hline & Técnico & & $0 \%$ \\
\hline & Profesional & 5 & $100 \%$ \\
\hline & Total & 5 & $100 \%$ \\
\hline \multirow{5}{*}{ Ocupación } & Psicologo & 1 & $20 \%$ \\
\hline & Administrador & $\mathbf{1}$ & $20 \%$ \\
\hline & Ing. Industrial & 2 & $40 \%$ \\
\hline & Contador & $\mathbf{1}$ & $20 \%$ \\
\hline & Total & 5 & $100 \%$ \\
\hline \multirow{6}{*}{$\begin{array}{c}\text { Cargo en la } \\
\text { empresa }\end{array}$} & Asist. Admon & 1 & $20 \%$ \\
\hline & Analista Mercad & 1 & $20 \%$ \\
\hline & Coord. Despach & 1 & $20 \%$ \\
\hline & Coord. Gestion & 1 & $20 \%$ \\
\hline & Jefe Contabilida & 1 & $20 \%$ \\
\hline & Total & 5 & $100 \%$ \\
\hline \multirow{3}{*}{ Estrato } & 3 & 3 & $60 \%$ \\
\hline & 4 & 2 & $40 \%$ \\
\hline & Total & 5 & $100 \%$ \\
\hline \multirow{5}{*}{$\begin{array}{c}\text { Años } \\
\text { trabajando } \\
\text { con la } \\
\text { empresa }\end{array}$} & 1,5 & 1 & $20 \%$ \\
\hline & 5 & 2 & $40 \%$ \\
\hline & 6 & 1 & $20 \%$ \\
\hline & 12 & 1 & $20 \%$ \\
\hline & Total & 5 & $100 \%$ \\
\hline \multirow{4}{*}{$\begin{array}{l}\text { Tipo de } \\
\text { vivien da }\end{array}$} & Propia & 1 & $20 \%$ \\
\hline & Arriendo & 3 & $60 \%$ \\
\hline & Familiar & 1 & $20 \%$ \\
\hline & Total & 5 & $100 \%$ \\
\hline Ciudad & Cali & 5 & \\
\hline contrato & Indefinido & 5 & \\
\hline Horas & 8 & 5 & \\
\hline
\end{tabular}

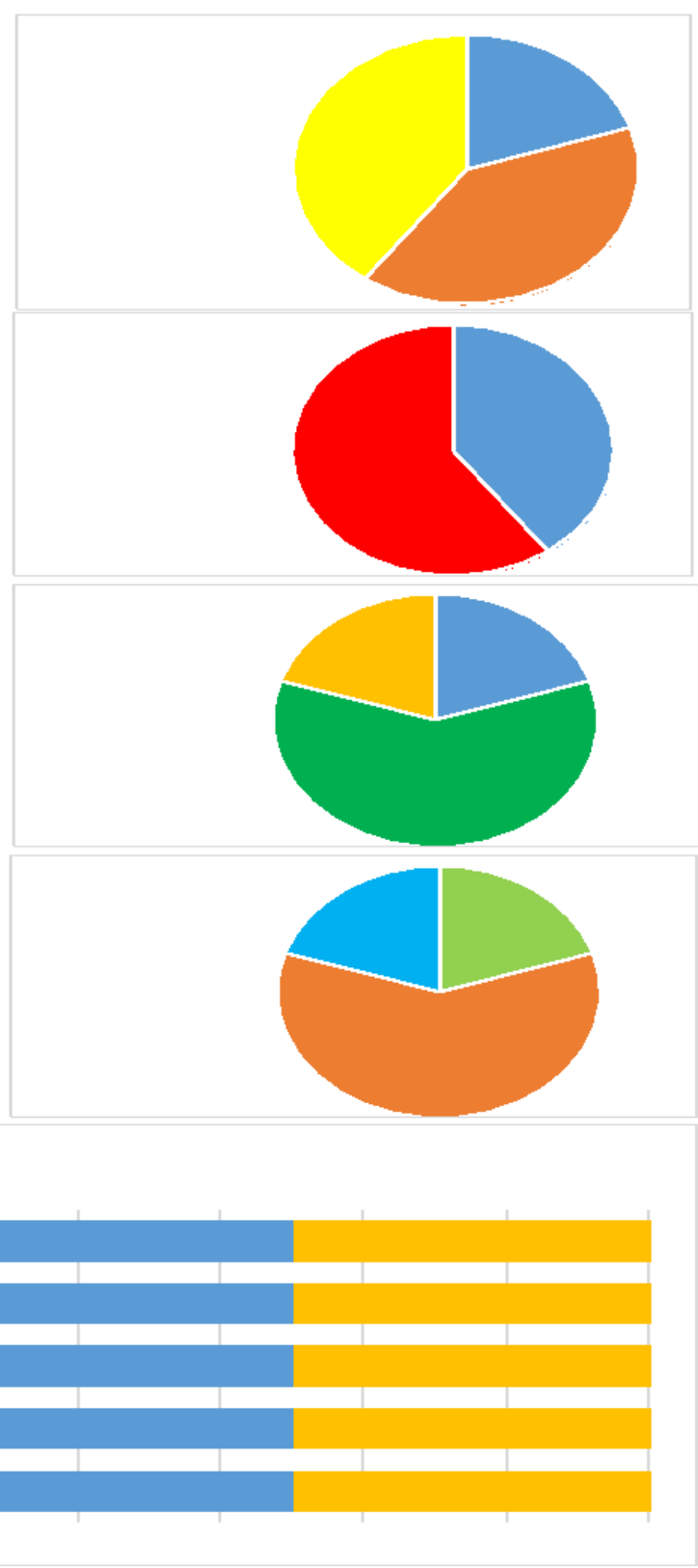


Fuente: Elaboración Propia.

Tal como indica la gráfica, el $60 \%$ de los encuestados son hombres y el $40 \%$ mujeres; de igual forma, todos los encuestados se encuentran entre el rango de edad de 20 a 50 Años; de los cuales el $40 \%$ está en el rango etario de 31 a 40 Años y otro $40 \%$ se encuentra entre 41 y 50 Años de edad y el 20\% restante son adultos jóvenes entre 20 y 30 años de edad.

El $60 \%$ pertenece al estrato socioeconómico 3 y el $40 \%$ al estrato 4 , de igual forma, el $80 \%$ están casados o en unión libre y solo el $20 \%$ se encuentra soltero. En cuanto a las características de la vivienda, el $20 \%$ afirma tener casa propia, seguido de un $60 \%$ afirma no tener casa propia y vivir de arriendo y el $20 \%$ que vive en casas familiares.

Todos los encuestados son profesionales, aunque no todos ocupan un cargo acorde a su nivel académico y su profesión, el $60 \%$ tienen cargos directivos y personal a cargo, como el jefe de Contabilidad, Coordinador de Gestión Humana y Coordinador de despachos y el otro 40\% se desempeña en Cargos Asistenciales y de apoyo.

Por otro lado todos los encuestados tienen contrato a término indefinido y el $80 \%$ lleva más de 5 años trabajando con la empresa y el 20\% lleva 1,5 años, situación que muestra estabilidad laboral en algunas áreas y cargos. 


\section{Resultado factores de riesgo extra laboral}

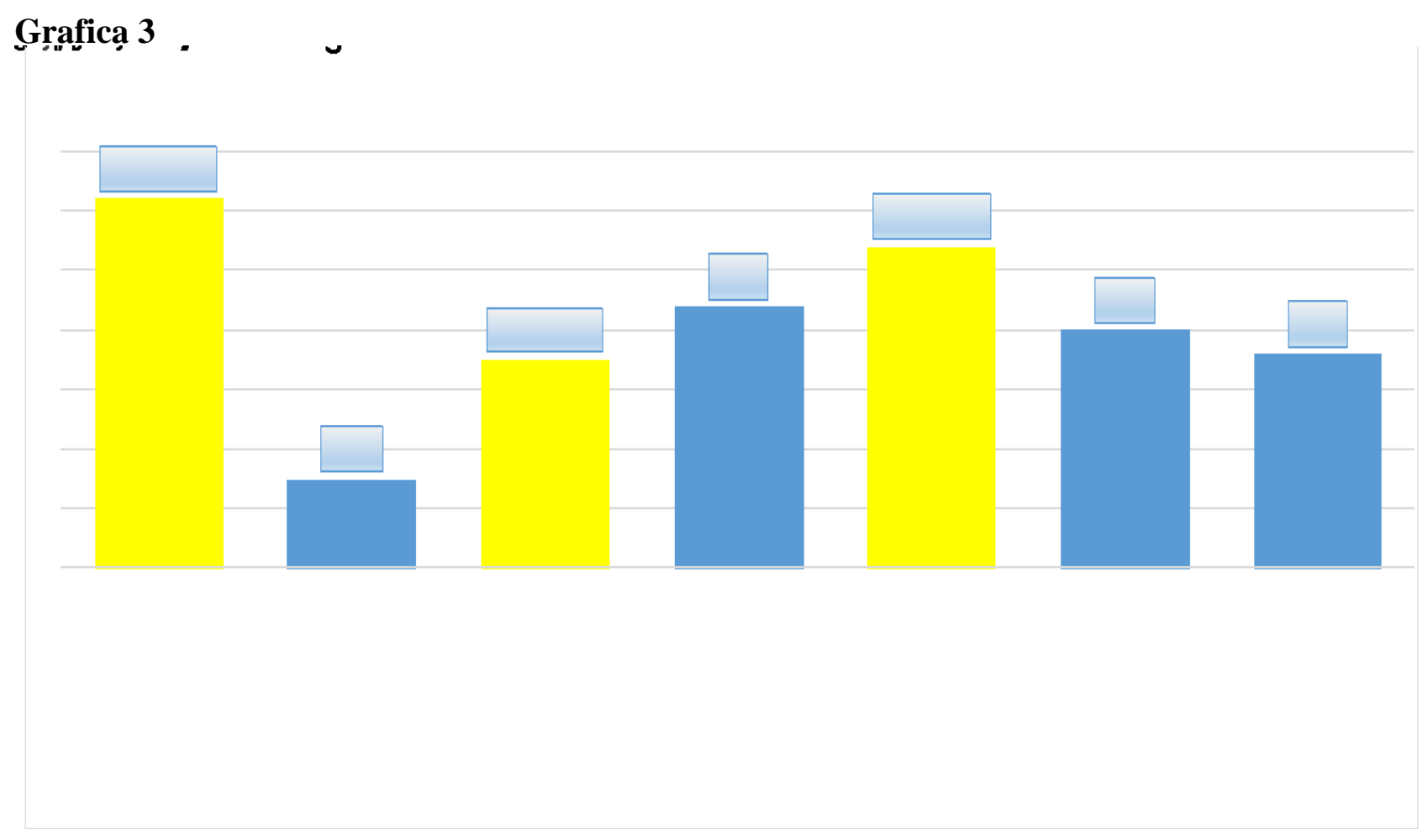

Fuente: Elaboración Propia.

Los factores de Riesgo Extra Laborales, se miden en 7 dimensiones que son:

Tiempo fuera del trabajo, Relaciones Familiares, Comunicación y Relaciones interpersonales, Situación económica del grupo familiar, Características de la vivienda y de su entorno, Influencia del entorno Extra laboral sobre el trabajo y Desplazamiento vivienda - trabajo-vivienda.

Se puede observar en la gráfica, tres de las dimensiones presentan un nivel de riesgo alto: La dimensión tiempo fuera del trabajo con 62 Puntos, Comunicación y Relaciones Interpersonales con 35 puntos y características de la vivienda y su entorno con 54 puntos.

Por otro lado aparecen otras dimensiones que aunque no representa un riesgo exageradamente alto, son relevantes porque su puntuación es alta: Situación económica del 
grupo familiar con un puntaje de 44, Influencia del entorno extra laboral sobre el trabajo con 40 puntos y desplazamiento de la vivienda al trabajo con 36 puntos. Finalmente aparece una dimensión que no representa riesgos, con 15 puntos que son las relaciones Familiares.

\section{Resultado nivel de estrés con sus patologías}

\section{Grafica 4}

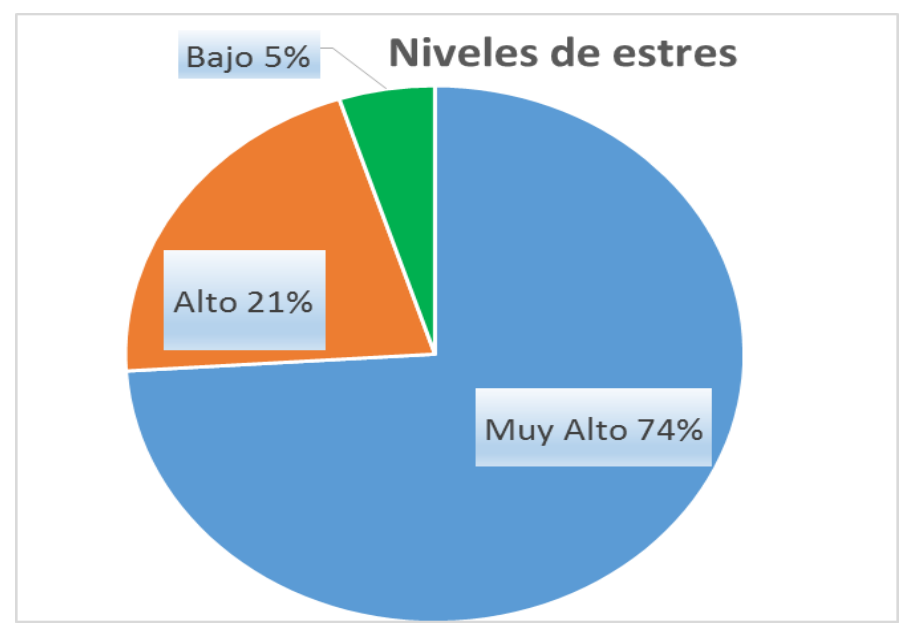

Fuente: Elaboración Propia.

El nivel de estrés arroja un resultado exagerado de un 74\% en "Muy alto", seguido de un $21 \%$ en "Alto" y un 5\% "Bajo" 


\section{Grafica 5}

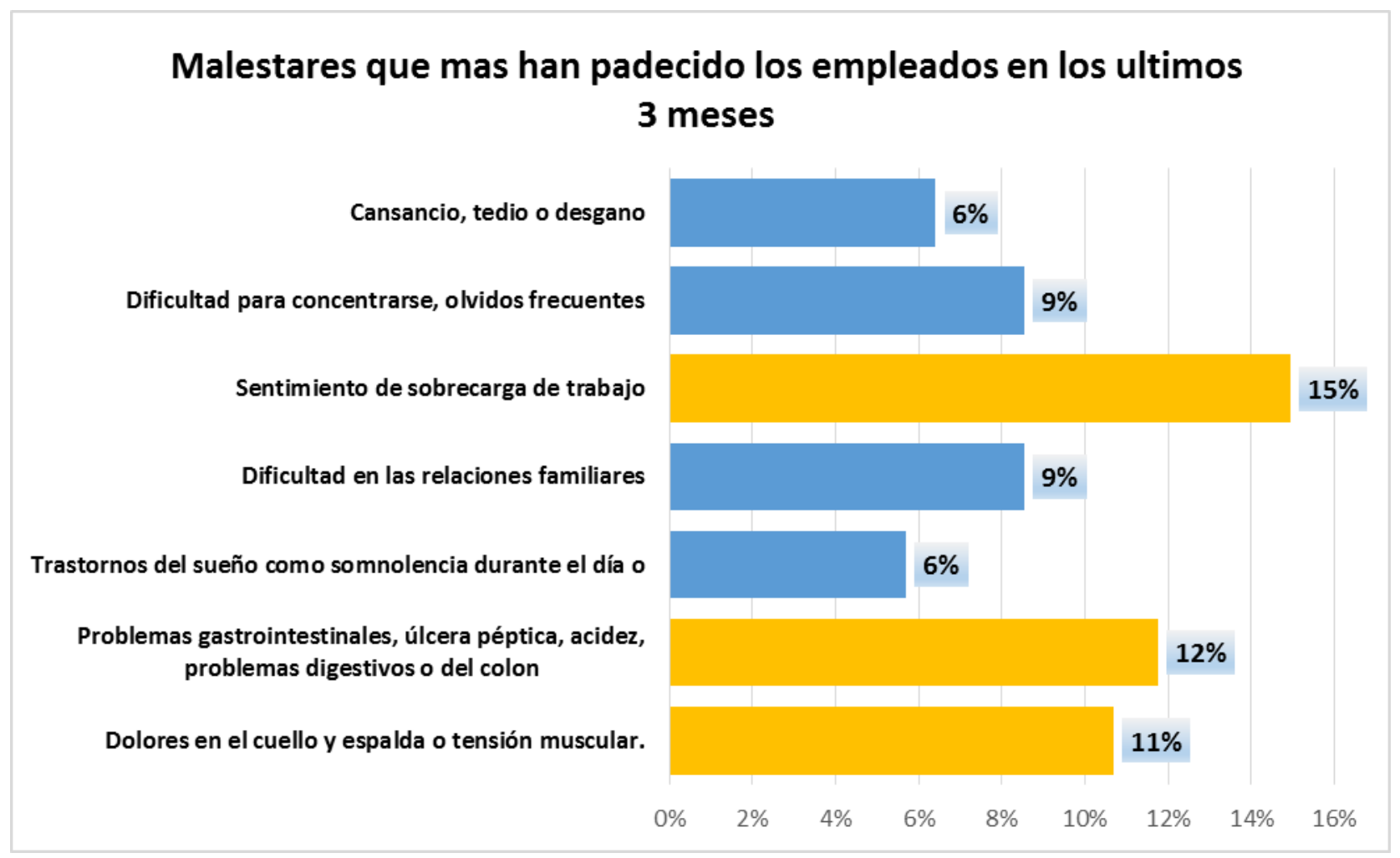

Fuente: Elaboración Propia.

En estos niveles de estrés, las patologías o malestares que más han presentado los encuestados durante los últimos tres meses son:

El $15 \%$ de los encuestados afirmaron mantener un sentimiento de sobrecarga de trabajo con un $15 \%$, seguido de problemas gastrointestinales como gastritis, colon irritable y reflujo etc. y un $12 \%$ que manifiesta haber tenido dolores de cuello, espalda o tensión Muscular 


\section{Análisis, interpretación y discusión resultados cualitativos}

Después de analizar la información recolectada en las encuestas las entrevistas y el ejercicio de observación, se encontró que la empresa Cosméticos, posee altos niveles de estrés en el $80 \%$ de la población encuestada; los cuales podrían estar relacionados con los factores de riesgo extra-laborales que arrojaron niveles altos de riesgo, de acuerdo a las dimensiones de la batería de factores de Riesgo Psicosociales del Ministerio de Protección Social (2010), quedando así:

Tabla 1: Elaboración Propia

\begin{tabular}{cl}
\hline Dimensiones & \multicolumn{1}{c}{ Factor de Riesgo } \\
\hline $\begin{array}{c}\text { Tiempo fuera del } \\
\text { trabajo. }\end{array}$ & $\begin{array}{l}\text { Se considera un riesgo, dado que los empleados de Cosméticos., } \\
\text { trabajan más de 8 horas diarias, y los fines de mes hasta 14 horas, y } \\
\text { además de eso se llevan trabajo para la casa, por lo tanto el empleado no } \\
\text { tiene tiempo para el ocio ni para compartir con su familia y amigos. }\end{array}$ \\
\hline $\begin{array}{c}\text { Situación Económica } \\
\text { del grupo Familiar. }\end{array}$ & $\begin{array}{l}\text { La mayoría de los encuestados afirman que lo que ganan en el núcleo } \\
\text { familiar no les alcanza para cubrir sus gastos básicos, que llevan 3 años } \\
\text { con el mismo salario, dado que las ventas y la productividad han bajado. }\end{array}$ \\
\hline $\begin{array}{c}\text { Comunicación y } \\
\text { Relaciones }\end{array}$ & $\begin{array}{l}\text { El } 60 \% \text { de los encuestados invierte parte de lo que gana en arriendo } \\
\text { porque no cuentan con casa propia. }\end{array}$ \\
$\begin{array}{c}\text { interpersonales. } \\
\text { familiar y social, son pobres, no por falta de habilidades de } \\
\text { comunicación; sino más bien por falta de tiempo por lo tanto se ha } \\
\text { creado distancia en estas redes de apoyo, que serían las que soportarían } \\
\text { al empleado en una crisis eventual. }\end{array}$ \\
$\begin{array}{cl}\text { Earacterísticas de la } 60 \% \text { de los encuestados pertenecen al estrato socioeconómico 3 y } \\
\text { viven en zonas alejadas al lugar de trabajo por lo que a algunos les toca } \\
\text { tomar dos transportes de ida y dos de venida para llegar al trabajo y a } \\
\text { vivienda se le suma que se demoran mucho en el desplazamiento, no solo por } \\
\text { la lejanía sino por los trancones. Por lo tanto se están privando de sueño }\end{array}$ \\
\hline
\end{tabular}


Por otro lado, según los resultados del cuestionario del MPS (2010) para la evaluación del estrés en su tercera edición arroja niveles altos de estrés, presentando las siguientes patologías; somnolencia, agotamiento, falta de energía dolores musculares, problemas gastrointestinales, sentimiento de sobrecarga de trabajo, dificultad para concentrarse, cansancio y ganas de no asistir al trabajo, disminución del rendimiento laboral, bajo compromiso con lo que hace, desinterés etc.

\section{Discusión}

Teniendo en cuenta que se trabajó con una muestra del 10\% de los empleados, se debe manejar con cautela los resultados, dado que se podría presentar un sesgo en la selección de la muestra; de igual forma, sería bueno contrastar la información, aplicando toda la batería de instrumentos del Ministerio de protección Social, dado que en este proyecto solo se tuvieron en cuenta los riesgos psicosociales extra laborales. Y se considera que algunos riesgos psicosociales intra laborales y las características de la personalidad de cada individuo, pueden estar influyendo en el estrés.

En el presente trabajo se muestran los resultados descriptivos de los factores de riesgo psicosocial extra laboral, niveles de estrés y patologías frecuentes, presentes en la empresa Cosméticos. Se presentan altos niveles de riesgos psicosociales extra laborales en varias dimensiones, los cuales se confirman con los niveles altos de estrés y con las patologías manifestadas por los empleados durante los últimos tres meses en los cuestionarios.

El resultado muestra que tres de las dimensiones presentan un nivel de riesgo Muy alto, que son: la dimensión tiempo fuera del trabajo con 62 puntos, comunicación y Relaciones Interpersonales con 35 puntos y características de la vivienda y su entorno con 54 puntos. Estas dimensiones que presentan un Nivel de riesgo Muy Alto, se asocia a niveles de 
respuesta muy altas de estrés, por lo tanto requieren intervención inmediata en el marco de un sistema de vigilancia epidemiológica.

Por otro lado aparecen otras dimensiones que aunque no representa un riesgo exageradamente alto, son relevantes porque su puntuación es Alta, esta categoría de riesgo también tiene una importante posibilidad de asociación con respuestas de estrés alto y por tanto, las dimensiones que se encuentren bajo esta categoría también requieren intervención en el marco de un sistema de vigilancia epidemiológica.

De igual forma es importante tener en cuenta la situación económica del grupo familiar con un puntaje de 44, Influencia del entorno extra laboral sobre el trabajo con 40 puntos y desplazamiento de la vivienda al trabajo con 36 puntos. Estas dimensiones presentan una respuesta moderada al estrés y ameritan observación y acciones preventivas.

Finalmente aparece una dimensión que no representa riesgos, con 15 puntos que son las relaciones Familiares. Esta dimensión no amerita desarrollar actividades de intervención pero si de promoción.

En Colombia, el MPS, expidió la resolución 2646 (2008), mediante la cual, "se establecen disposiciones y se definen responsabilidades para la identificación, evaluación, prevención, intervención y monitoreo permanente de la exposición a factores de riesgo psicosociales y para la determinación del origen de las patologías causadas por el estrés ocupacional" (p.1).

Sin embargo, muchas empresas no le dan importancia a las condiciones del entorno familiar social y económico del trabajador y se limitan a prevenir los riesgos Psicosociales dentro de la empresa y luego se preguntan, porque a pesar de las estrategias preventivas los empleados se siguen ausentando e incapacitando por enfermedades psicosomáticas, físicas y otros malestares relacionados al estrés. 
LaMontagne, mencionado por Weir, (2013) afirma que "los empleadores, deben tener en cuenta los factores de riesgo relacionados con la salud mental, independientemente de su causa, dado que al final no importa si el empleado está deprimido por causas laborales o por problemas familiares" (parr.12); porque de todas formas esa patología le va a afectar su rendimiento laboral y su salud física y mental.

Es decir, que un empleado traslada su estrés familiar y social hacia su lugar de trabajo sumándose al estrés normal del trabajo y luego, sale para su casa cargado con el estrés que llegó sumado al estrés laboral, no duerme bien y al día siguiente, lo mismo, convirtiéndose en un ciclo vicioso que deteriora el organismo y la salud mental.

Lo cual se corrobora con lo que afirma la OIT/OMS en el informe de Comité mixto sobre factores psicosociales (1984).

El tiempo en el lugar de trabajo, cambia la dinámica familiar y social y la forma de vida afectando la salud del trabajador (...) (p.16), a lo que se suma el desempleo y la situación económica, dado que afecta la estabilidad laboral y por ende el bienestar de los trabajadores que aceptan trabajar en condiciones inhumanas con horarios extendidos y una carga laboral alta, colocando al trabajador en una posición débil para enfrentar la carga laboral (...) (p.20). Esta rutina diaria, a mediano plazo, trae consecuencias negativas a nivel psicológico, dado que deteriora la relación con su familia y amigos llegando a casa cansado y de mal genio, lo cual le puede generar insomnio, irritación, preocupación, tensión y depresión; actitudes negativas que transportará de su casa al trabajo derivándose en problemas cognoscitivos como pérdida de capacidad de atención, memoria y toma de decisiones (p.25), generando estrés. (p. 6-25)

Entre los malestares que manifestaron los encuestados haber padecido durante los últimos 3 meses, se encuentran: sentimiento de sobrecarga de trabajo con un $15 \%$, seguido de problemas gastrointestinales como gastritis, colon irritable y reflujo etc. y un $12 \%$ que 
manifiesta haber tenido dolores de cuello, espalda o tensión muscular. Todos estos malestares son consecuencia de la exposición frecuente a altos niveles de estrés

El Decreto 1477, adopta la nueva tabla de enfermedades laborales con el fin de mejorar las condiciones del empleado; sin embargo en esta tabla no se especifica claramente el estrés (...), pero si mencionan unas patologías causadas por la exposición continua a factores de riesgo Psicosociales, entre las cuales se incluyen: gastritis, ansiedad, trastornos emocionales, de comportamiento y de adaptación; síndrome del colon irritable, trastornos del sueño, de igual forma depresión, Infarto del miocardio y otras urgencias cardiovasculares, hipertensión arterial etc. (p.2628).

Aunque este decreto, no especifica claramente que estas patologías son causadas por estrés, varios estudios a nivel internacional afirman que estas se dan por la exposición continua al estrés; por lo tanto; se deduce que estas patologías son causadas por estrés (Ver anexo 3 Tabla de enfermedades laborales)

Los factores externos al sitio de trabajo, no están bien atendidos por los empresarios, representando un factor de riesgo alto para la salud del empleado; porque "cualquier circunstancia familiar o de la vida personal, de la cultura, de la nutrición, ó de las facilidades de transporte y vivienda, pueden generar estrés" (OIT/OMS, 1994, p.112

Se debe tener en cuenta estos factores de riesgo fuera del trabajo, dado que la exposición continua a altos niveles de estrés generan desgaste de energía, y agotamiento emocional que repercuten en la salud del trabajador desarrollando enfermedades físicas como gastritis, reflujo, dolores lumbares y musculares, dolores de cabeza y enfermedades psicosomáticas como sentimientos de ineficacia y baja autoestima, falta de sueño, falta de apetito, apatía, dolores crónicos etc. 


\section{Referencias Bibliograficas}

Aguilera Castro A. (2012) Crecimiento empresarial, basado en la responsabilidad social corporativa. Scielo 1(26), 1-26. Recuperado de: http://www.scielo.org.co/pdf/pege/n32/n32a02.pdf

Betancur Gómez, FM (2013) Factores Psicosociales en el trabajo, Revista Gestión Humana Ed. 8 Publicaciones Legis S.A. (p.28)

Benavides, F. G., Gimeno, D., Benach, J., Martínez, J. M., Jarque, S., Berra, A., y Devesa, J. (2002). Descripción de los factores de riesgo psicosocial en cuatro empresas. Gaceta sanitaria, 16(3), 222-229. Recuperado de http://www.scielosp.org/pdf/gs/v16n3/v16n3a02.pdf

Comisión Europea (1999) Guía sobre el estrés relacionado con el trabajo, recuperado de: http://www.srt.gob.ar/Super/eventos/2004/BancoNacion/contenido/1 guiasobreelestr es.pdf

Decreto 1832 de 1994 Tabla de enfermedades Profesionales, Articulo 1, Punto 42, (p.5) recuperado de: http://www.alcaldiabogota.gov.co/sisjur/normas/Norma1.jsp?i=8802

Decreto 477 de 2014 Tabla de enfermedades profesionales, Sección I Agentes Psicosociales (p. 26). Recuperado de: http://www.alcaldiabogota.gov.co/sisjur/normas/Norma1.jsp?i=36783

Hernández, R., Fernández, C. y Baptista, P. (2003). Metodología de la investigación (3a ed.). México: Editorial Mc Graw-Hill. 
Ministerio de la Protección Social. (2007). Informe final de encuesta nacional de condiciones de Salud y Trabajo en el Sistema General de Riesgos Profesionales (I ENCST) (p.95). Recuperado el 27 de marzo de 2016 de: http://www.oiss.org/estrategia/IMG/pdf/I_encuesta_nacional_colombia2.pdf

Ministerio de Protección Social. (2007). Protocolo para la determinación del origen de las patologías derivadas del estrés. Pontificia Universidad Javeriana. Bogotá. http://fondoriesgoslaborales.gov.co/Noticias/Noticia.aspx?IdNoticia=154

Ministerio de la Protección Social. (2008). Resolución 2646 de julio de 2008 - establece Disposiciones y se definen responsabilidades para la identificación, evaluación y prevención de factores Psicosociales en el trabajo: (p.1). Recuperado el 25 de marzo de 2016 de:

http://copaso.upbbga.edu.co/legislacion/resolucion_2646_2008_Factores\%20de\%20 $\underline{\text { Riesgo\%20Sicosocial.pdf }}$

Ministerio de la Protección Social. "Batería de instrumentos para la evaluación de factores de riesgo psicosociales”. (2010). Pontificia Universidad Javeriana. Bogotá. Recuperado de: http://fondoriesgoslaborales.gov.co/Contenido/Default.aspx? $\mathrm{Id}=568$

OIT-OMS. Organización Internacional del Trabajo-Organización Mundial de la Salud. Factores psicosociales en el trabajo: naturaleza, incidencia y prevención. Informe del comité mixto OIT/OMS sobre medicina del trabajo. Ginebra: OIT, 1984. Recuperado de: http://biblioteca.uces.edu.ar/MEDIA/EDOCS/FACTORES_Texto.pdf 
Organización Mundial de la Salud (2013), Un estado de bienestar (Mensaje en un blog), recuperado de: http://www.who.int/features/factfiles/mental health/es/

Villalobos, G., Vargas, A., Escobar, J., Jiménez, M., Rondón, M. (2010). Batería de instrumentos para la evaluación de factores de riesgo psicosocial. Ministerio de la Protección Social. Primera edición

Weir, Kirsten (September 2013) Work, stress and health, 10th International Conference on Occupational Stress and health in los Angeles by the American Psychological Association (APA). Recuperated from: http://www.apa.org/monitor/2013/09/stresshealth.aspx 